\title{
Arthroscopic Fixation of Tibial Intercondylar Eminence Fracture by Fiber Wire Suture and U Screws in Adolescent Athletes
}

\author{
Murat Çalbıyık $^{1}$, Tuba Denizci ${ }^{2}$ \\ ${ }^{1}$ Hitit University Medicine Faculty Department of Orthopaedic and Traumatology Çorum, Turkey \\ ${ }^{2}$ Hitit University, Faculty of Sports Science, Corum, Turkey
}

Received: 4 August 2017, Accepted 26 August 2017, Published online: 28 December 2017

(C) Ordu University Institute of Health Sciences, Turkey, 2017

\begin{abstract}
Objective: We aimed to present our experience on arthroscopic management of tibial intercondylar eminence fracture in adolescent athletes.

Methods: This was a case series of 9 adolescents ( 7 males, 2 females) aged 10-16 years diagnosed with tibial intercondylar eminence fracture following a sports activity. According to Meyers and McKeever classification, 4 patients had Type IIIA, 3 had Type IIIB, and 2 patients had Type II fracture. Following percutaneous arthroscopic reduction, the fragments of the fracture were fixed internally and anterior cruciate ligament was reconstructed through two $2.4 \mathrm{~mm}$ parallel tunnels at the level of anterior tibia by using fiber wire suture and U screws. Anterior tibial displacement was measured by KT-1000 knee arthrometer, and Lysholm Knee Scoring Scale was used for the patient-reported outcomes.

Results: The duration of operation was $31.6 \pm 8.9 \mathrm{~min}$. The patients were followed up for $37.1 \pm 19.1$ months. Theanterior tibial displacement relative to the femur was $1.4 \pm 0.7 \mathrm{~mm}$. TheLysholm score was $95.8 \pm 3.1$. None of the patients developed infection. The complete fracture healing was obtained in all patients.

Conclusion: Arthroscopic reduction and internal fixation by fiber wire suture and $\mathrm{U}$ screws provide perfect anatomic and functional outcome for displaced Type II and Type III tibial intercondylar eminence fracture in adolescent athletes.

Key words: Adolescent, sport injury, knee joint, arthroscopic reduction, tibial intercondylar eminence fracture

Address for correspondence/reprints:

Murat Çalbıyık

Telephone number: +90 (532)7698962

E-mail: drmuratcalbiyik@ @otmail.com

DOI: $10.19127 / \mathrm{mbsjohs} .332789$

\section{Introduction}

Sports injuries causing fractures around epiphyseal and apophyseal knee joint is common in pediatric population (Beaty and Kumar, 1994). Among these fractures, the tibial intercondylar eminence fracture is mostly seen in children with an incidence of 3/100,000/year due to incomplete tibial epiphyseal bone and weak nature of anteriour cruciate ligament in skeletally immature population (Leeberg et al. 2014). This fracture has been classified by Meyers and McKeever into three groups according to degree of displacement-Type I, Type II and Type III (Meyers and McKeever, 1959; Meyers and McKeever, 1970).
\end{abstract}


The tibial intercondylar eminence fracture is relatively rare compared to other common pediatric fractures, thus underdiagnosed; however, when untreated it may result in knee instability and disruption of physis (Leeberg et al. 2014). Although there is no consensus on whether surgery is needed for Type II and III fractures (Molander et al. 1981), arthroscopic surgery is the preferred method of treatment in recent literature (Leeberg et al. 2014; Owens et al. 2003). Compared to open surgery, arthroscopical management of tibial intercondylar eminence fracture is less invasive and provides earlier mobilization (Leeberg et al. 2014; Larsen et al. 2006). However, many different surgical techniques using various sutures and screws have been used during arthroscopic surgery. Therefore, there is a disagrement on the surgical technique for the management of displacedtibial intercondylar eminence fracture. Furthermore, there is a continuous debate on whether to cross physis during fixating the fracture. Therefore, there is still need for more experience on various surgical techniques in order to reach a consensus on the management of this critical fracture.

In the present study, we aimed to present our experience on arthroscopic management of tibial intercondylar eminence fracture in adolescent athletes.

\section{Methods}

\section{Study design and population}

This was a case series of 9 adolescent athelets ( 7 males, 2 females) aged 10-16 years who were diagnosed with tibial intercondylar eminence fracture following a sports activity and treated arthroscopically in Hitit University Faculty of Medicine Department of Orthopedics and Traumatology between May 2010 and May 2016. The fractures were classified according to Meyers and McKeever classification (Meyers and McKeever, 1959: Meyers and McKeever, 1970). The patients and parents were informed about the treatment, and legal represantative of each patient gave written consent before the surgical operation. The study was conducted in accordance to the latest version of Helsinki Declaration.

\section{Arthroscopic procedure and postoperative follow-up}

Following percutaneous arthroscopic reduction, the fragments of the fracture was fixed internally and anterior cruciate ligament was reconstructed through two 2.4-mm parallel tunnels at the level of anterior tibia by using fiber wire suture and $U$ screws. On postoperative period, after confirming the stability of the fracture fragments by arthroscopy and C-arm scopy, the knee was immobilized in a brace locked in full extension for 6 weeks. Isometric quadriceps and hamstring exercises were started 2 days after the operation. Partial weight-bearing was allowed after two weeks. The knee brace was removed after 6 weeks, and rehabilitation and strengthening exercises were started. The patients were allowed to make sportive activities after 5 months.

The fracture was evaluated before and after the operation by X-ray, conventional and threedimensional computed tomography (CT), and magnetic resonance (MR) imaging. On postoperative 12th month, anterior tibial displacement relative to the femur was measured by KT-1000 knee arthrometer (KT-1000, MedMetric Co. San Diego, California, USA) (Arneja and Leith, 2009). For the patient-reported outcomes of the surgery, Lysholm Knee Scoring Scale that measures the effect of knee problem on patient's daily life on a 0 to 100 scale was used (Lysholm and Gillquist, 1982).

The study data were reported using descriptive statistics (e.g., frequency, percentage, mean, standard deviation).

\section{Results}

The tibial intercondylar eminence fracture occurred after cycling in 4 patients, wrestling in 3 patients, basketball in 1 patient, and skiing in 1 patient. The fracture was in right knee in 4 patients and left knee in 5 patients. On the basis of Meyers and McKeever classification, 4 patients had Type IIIA, 3 patients had Type IIIB and 2 patients had Type II fracture. The anterior cruciate ligament rupture was present together with eminence fracture in 3 patients. The clinical characteristics of patients were summarized in Table 1.

The mean duration of operation was $31.6 \pm 8.9$ min (Table 2). The patients were followed up for $37.1 \pm 19.1$ months on average after the arthroscopic procedure. There was a minimum anterior tibial displacement relative to the femur as measured by 
the KT-1000 knee arthrometer, which was $1.4 \pm 0.7$ $\mathrm{m}$ on average. A mean high Lysholm score, which was $95.8 \pm 3.1$, indicated a favorable patient-reported outcome of the surgical intervention (Table 2). None of the patients developed infection. The complete fracture healing was recorded in control radiographs of all patients. The pre- and postoperative images of a 12-year old male patient were present in Figure 1 and 2 , respectively.

Table 1. Demographic and clinical characteristics of study patients $(\mathrm{n}=9)$

\begin{tabular}{|c|c|}
\hline Variable & Result \\
\hline $\begin{array}{l}\text { Age (years), mean } \pm \text { standard deviation } \\
\text { (range) }\end{array}$ & $12.44 \pm 2.0(10-16)$ \\
\hline \multicolumn{2}{|l|}{ Gender, n (\%) } \\
\hline Male & $7(77.8 \%)$ \\
\hline Female & $2(22.2 \%)$ \\
\hline \multicolumn{2}{|l|}{ Side of eminence fracture, $\mathrm{n}(\%)$} \\
\hline Right knee & $4(44.4 \%)$ \\
\hline Left knee & $5(55.6 \%)$ \\
\hline \multicolumn{2}{|l|}{ Sport activity causing fracture, $\mathrm{n}(\%)$} \\
\hline Wrestling & $3(33.3 \%)$ \\
\hline Basketball & $1(11.1 \%)$ \\
\hline Skiing & $1(11.1 \%)$ \\
\hline Cycling & $4(4.4 \%)$ \\
\hline \multicolumn{2}{|l|}{$\begin{array}{l}\text { Meyers and McKeever classification } \\
\text { of fracture, } \mathrm{n}(\%)\end{array}$} \\
\hline Type IIIA & $4(44.4 \%)$ \\
\hline Type IIIB & $3(33.3 \%)$ \\
\hline Type II & $2(22.2 \%)$ \\
\hline \multicolumn{2}{|l|}{ Concomittant injuries, $\mathrm{n}(\%)$} \\
\hline None & $4(44.4 \%)$ \\
\hline Anterior cruciate ligament rupture & $3(33.3 \%)$ \\
\hline Radius fracture & $1(11.1 \%)$ \\
\hline Elbow fracture & $1(11.1 \%)$ \\
\hline Variable & $\begin{array}{c}\text { Mean } \pm \text { standard } \\
\text { deviation }\end{array}$ \\
\hline $\begin{array}{l}\text { Duration of operation ( } \mathrm{min} \text { ) } \\
\text { Postoperative follow-up duration } \\
\text { (months) }\end{array}$ & $37.1 \pm 19.1$ \\
\hline KT-1000 knee arthrometer & $1.4 \pm 0.7$ \\
\hline Lysholm score & $95.8 \pm 3.1$ \\
\hline
\end{tabular}

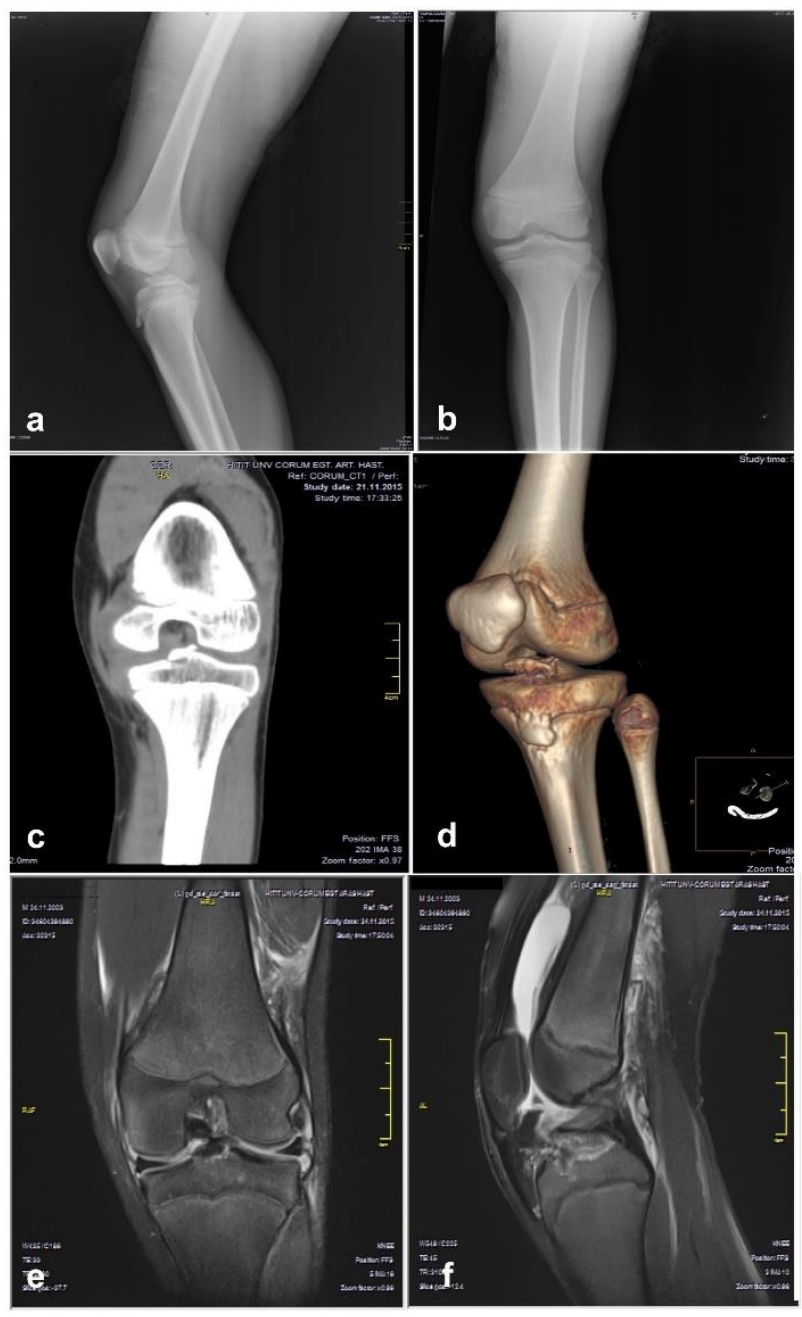

Figure 1. Preoperative radiologic images of 12-year old male patient with Type IIIA tibial intercondylar eminence fracture. a, lateral X-ray image; b, anteroposterior X-ray image; c, coronal CT image; $d$, three-dimensional CT image; e, coronal MR image; $f$, sagittal MR image. 

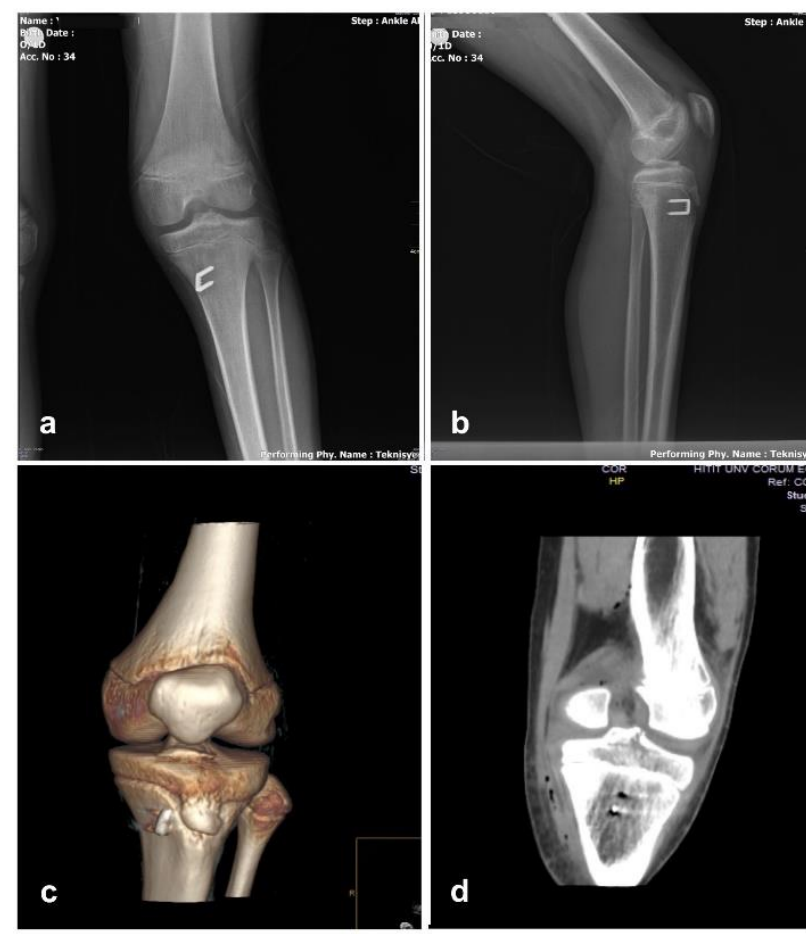

Figure 2. Postoperative images of the patient in Figure 1 a, anteroposterior X-ray image; b, lateral X-ray image; c, three-dimensional CT image; $d$, coronal CT image.

\section{Discussion}

In this case-series, we report our experince with arthroscopic reduction and internal fixation by fiber wire suture and U screws for displaced Type II and Type III tibial intercondylar eminence fracture in 9 adolescent athelets. We obtained good anatomic and functional outcomes without any postoperative complication over 3-year postoperative follow-up duration.

As the compatitive sport activities became popular in adolescents, particularly in males, sport injuries affecting knee are frequently encountered in this age group. The treatment approach against knee injuries at this period has critical importance for the need to preserve both functionality of the knee and the integrity of the physis (Schmittenbecher, 2005). In etiology, the tibial intercondylar eminence fracture is equivalent to the ruptures of the anterior cruciate ligament, thus anatomic reduction is needed to preserve the stability of the joint.

The prognosis is basicly related to the type of fracture, anatomic reduction, articular congruity, and age of patient (Tudisco et al. 2010). Majority of the previous studies suggest that Type I fractures and those in children below 10 years of age can be treated conservatively, which is the closed reduction and immobilization in extension (Tudisco et al. 2010;
Atay et al. 2002; Wilfinger et al. 2009). Although some studies suggested that conservative treatment is justified in most cases of Type III tibial intercondylar eminence fracture in children (Molander et al. 1981), for displaced fractures, longterm results were not satisfactory thus surgical treatment should be applied (Atay et al. 2002; Casalonga et al. 2010). Although some recent reports showed very favorable outcome with open reduction and cross wire fixation (Keshet et al. 2015), in comparison to open surgical techniques, arthroscopic reduction and internal fixation showed overall better results (Tudisco et al. 2010; Prince and Moyer, 1995).

For the arthroscopic management of Type III fracture, various techniques were suggested including the usage of the Kirschner wires, Arthrex suture lasso device, folded surgical steels, arthroscopy-guided intra-articular button fixation, Herbert-screw fixation, headless compression screw, and cannulated screw (Su et al. 2011; Oohashi, 2001; Memisoglu et al. 2016; Wiegand et al. 2014; Johnson and Durbin, 2012; Furlan et al. 2010; Senekovid and Veselko, 2003), all of which revealed good functional outcome in short- and long-term. Recent improvements in suture materials provided that sutures with an improved fixation technique are as efficient as cannulated screws (Memisoglu et al. 2016; Wiegand et al. 2014; Johnson and Durbin, 2012). In the present case series, we applied internal fixation by fiber wire suture and $U$ screws and obtained good anatomic and functional outcome assessed by KT-1000 knee arthrometer and Lysholm score.

Most of the previous studies on the arthroscopic management of tibial intercondylar eminence fracture in literature are case-reports or retrospective series. One of the largest reports published recently by Persiani et al. (2016) was a retrospective analysis of 41 adolescent athletes who were treated successfully by conservative approach, open surgery or arthroscopy depending on severity of knee injury. In their study, Persiani et al. (2016) suggested performing a CT exam to exclude an intra-articular physeal fracture in this age group of patients. In our series, pre- and postoperative evaluations were performed by X-ray, conventional and threedimensional CT, and MR imaging.

Many previous studies used arthroscopy to evaluate the outcomes after surgery (Leeberg et al. 2014). Similarly, we performed arthroscopy and Carm scopy for postoperative confirmation of 
fixation. For long-term follow-up we used control radiographs.

The most common complications of knee arthroplasty in childhood is comminution of the fracture fragments, nonunion, infection, joint instability, stiffness, laxity, extension impingement, growth disturbances, and meniscus lesion (Johnson and Durbin, 2012; Kieser et al. 2011). None of the patient in our series developed any intra- or postoperative complication.

The main limitations of the present study are its small sample size and retrospective design, which preclude us from reaching a definitive conclusion on the surgical technique during arthroscopical treatment of displaced tibial intercondylar eminence fractures in skeletally immature children. On the basis of case-reports and case-series including the present report on various surgical techniques, further larger scale and prospective studies with longer follow-up duration are needed.

\section{Conclusion}

In conclusion, arthroscopic reduction and internal fixation by fiber wire suture and $\mathrm{U}$ screws provide perfect anatomic and functional outcome for displaced Type II and Type III tibial intercondylar eminence fracture in adolescent athelets. The displaced tibial intercondylar eminence fracture should be treated with arthroscopic reduction and internal fixation. On the basis of our experince, we believe that since fiber wire sutures provide improved fixation in the arthroscopic management of tibial intercondylar eminence fracture in children, they should be preferred to screws.

Ethics Committee Approval: The requirement for the ethics committee approval was waived for the retrospective design and valid legal regulations at the time of the study.

Peer-review: Externally peer-reviewed.

Author Contributions: Idea- M.Ç.; Design M.Ç; Supervision- M.Ç.; Funding- M.Ç; Materials- M.Ç.; Data Collection/Data Process- M.Ç., T.D., Analyze or Comment- M.Ç, T.D., Literature Scanning- M.Ç., T.D.; Writer of Paper- M.Ç., T.D.; Critical ReviewM.Ç.

Conflict of Interest: No conflict of interest was declared by the author.

Financial Disclosure: The authors declared that this study has received no financial support.

\section{References}

Arneja S, Leith J. Review article: Validity of the KT1000 knee ligament arthrometer. Journal of Orthopedic Surgery (Hong Kong), 2009; 17: 7779.

Atay OA, Doral MN, Tetik O, Leblebicioğlu G. Conservative treatment of eminentia intercondylaris fractures of the tibia in children. Turkish Journal of Pediatrics, 2002: 44: 142-145.

Beaty JH, Kumar A. (Fractures about the knee in children. Journal of Bone and Joint Surgery, 1994; 76: 1870-1880.

Casalonga A, Bourelle S, Chalencon F, De Oliviera L, Gautheron V, Cottalorda J. Tibial intercondylar eminence fractures in children: The long-term perspective. Orthopaedics \& Traumatology: Surgery \& Research, 2010; 96: 525-530.

Furlan D, Pogorelić Z, Biocić M, Jurić I, Mestrović J. Pediatric tibial eminence fractures: arthroscopic treatment using K-wire. Scandinavian Journal of Surgery, 2010; 99: 3844.

Johnson DL, Durbin TC. Physeal-sparing tibial eminence fracture fixation with a headless compression screw. Orthopedics, 2012; 35: 604608.

Keshet D, Zaidman M, Eidelman M. Treatment of avulsion fractures of the intercondylar eminence by medial parapatellar approach, open reduction and cross wire fixation. Journal of Pediatric Orthopaedics B, 2015; 24: 321-325.

Kieser DC, Gwynne-Jones D, Dreyer S. Displaced tibial intercondylar eminence fractures. Journal of Orthopaedic Surgery (Hong Kong), 2011; 19: 292-296.

Larsen MW, Garrett WE, Delee JC. Surgical management of anterior cruciate ligament injuries in patients with open physes. Journal of American Academy of Orthopaedic Surgeons, 2006; 14: 736-744.

Leeberg V, Lekdorf J, Wong C, Sonne-Holm S. Tibial eminentia avulsion fracture in children - a systematic review of the current literature. Danish Medical Journal, 2014; 61: A4792.

Lysholm J, Gillquist J. Evaluation of knee ligament surgery results with special emphasis on use of a scoring scale. American Journal of Sports Medicine, 1982; 10: 150-154. 
Memisoglu K, Muezzinoglu US, Atmaca H, Sarman H, Kesemenli CC. Arthroscopic fixation with intra-articular button for tibial intercondylar eminence fractures in skeletally immature patients. Journal of Pediatric Orthopaedics B, 2016; 25: 31-36.

Meyers MH, McKeever FM. Fracture of the intercondylar eminence of the tibia. Journal of Bone and Joint Surgery - American Volume, 1970; 52: 1677-1684.

Meyers MH, McKeever FM. Fracture of the intercondylar eminence of the tibia. Journal of Bone and Joint Surgery - American Volume, 1959; 41-A: 209-222.

Molander ML, Wallin G, Wikstad I. Fracture of the intercondylar eminence of the tibia: a review of 35 patients. Journal of Bone and Joint Surgery British Volume, 1981; 63-B: 89-91.

Oohashi Y. A simple technique for arthroscopic suture fixation of displaced fracture of the intercondylar eminence of the tibia using folded surgical steels. Arthroscopy, 2001; 17: 10071011.

Owens BD, Crane GK, Plante T, Busconi BD. Treatment of type III tibial intercondylar eminence fractures in skeletally immature athletes. American Journal of Orthopedics, 2003; 32: 103-105.

Persiani P, Ranaldi FM, Formica A, Mariani M, Mazza O, Crostelli M, Villani C. Apophyseal and epiphyseal knee injuries in the adolescent athlete. Clinical Therapeutics, 2016; 167: e155-e161.

Prince AR, Moyer RA. Arthroscopic treatment of an avulsion fracture of the intercondylar eminence of the tibia. Case report. American Journal of Knee Surgery, 1995; 8: 114-116.

Schmittenbecher PP. What must we respect in articular fractures in childhood? Injury 2005; 36: A35-43.

Senekovic V, Veselko M. Anterograde arthroscopic fixation of avulsion fractures of the tibial eminence with a cannulated screw: five-year results. Arthroscopy, 2003; 19: 54-61.

Su WR, Wang PH, Wang HN, Lin CJ. A simple, modified arthroscopic suture fixation of avulsion fracture of the tibial intercondylar eminence in children. Journal of Pediatric Orthopaedics B, 2011; 20: 17-21.

Tudisco C, Giovarruscio R, Febo A, Savarese E, Bisicchia S. Intercondylar eminence avulsion fracture in children: long-term follow-up of 14 cases at the end of skeletal growth. Journal of Pediatric Orthopaedics B, 2010; 19: 403-408.
Wiegand N, Naumov I, Vámhidy L, Nöt LG. Arthroscopic treatment of tibial spine fracture in children with a cannulated Herbert screw. Knee, 2014; 21: 481-485.

Wilfinger C, Castellani C, Raith J, Pilhatsch A, Höllwarth ME, Weinberg AM. Nonoperative treatment of tibial spine fractures in children-38 patients with a minimum follow-up of 1 year. Journal of Orthopaedic Trauma, 2009; 23: 519524. 